\title{
Simultaneous Determinations of Methoxylated Polybrominated Diphenyl Ethers and Polybrominated Diphenyl Ethers in Dairy Products from China by GC-MS
}

\author{
Decong $\mathrm{Hu}^{1,2,{ }^{*}}$, Xiaozhong $\mathrm{Hu}^{1,2}$, Wei Chen ${ }^{1,2}$, Hong Wang ${ }^{3}$, Kuanzheng $\mathrm{Zhu}^{3}$, Bin Wu ${ }^{4}$, \\ Changjun Lin ${ }^{4}$ \\ ${ }^{1}$ Hubei Entry-Exit Inspection and Quarantine Bureau of PRC, Wuhan, China \\ ${ }^{2}$ China Certification \& Inspection Group HuBei Co., Ltd, Wuhan, China \\ ${ }^{3}$ Hubei Provincial Supervision and Inspection Research Institute for Products Quality, Wuhan, China \\ ${ }^{4}$ Technology Center of Liaoning Entry-Exit Inspection and Quarantine Bureau of PRC, Changjiang Dalian, China
}

Email address:

deconghu_hust@163.com (Decong Hu)

\section{To cite this article:}

Decong Hu, Xiaozhong Hu, Wei Chen, Hong Wang, Kuanzheng Zhu, Bin Wu, Changjun Lin. Simultaneous Determinations of Methoxylated Polybrominated Diphenyl Ethers and Polybrominated Diphenyl Ethers in Dairy Products from China by GC-MS. Science Journal of Analytical Chemistry. Vol. 3, No. 6, 2015, pp. 80-90. doi: 10.11648/j.sjac.20150306.12

\begin{abstract}
In this paper, the method of simultaneous determinations of methoxylated polybrominated diphenyl ethers and polybrominated diphenyl ethers in dairy products from China was investigated. PBDEs are widely used brominated flame retardant, which are increasingly reported in the environment. MeO-PBDEs are structural analogs to PBDEs, and reported as natural products and novel pollutants present in the environment. A new isotopic dilution GC-MS method was firstly developed to simultaneously determine fourteen PBDEs and eight MeO-PBDEs in dairy products (full cream milk powder, skim milk powder, pure milk and acidophilus milk) in this study. Solvent extraction, gel permeation chromatography (GPC) and silica gel column cleanup were used, some important steps and crucial parameters were modified and intensified compared with other literatures. Besides, the conditions of GC and MS were also optimized. The limits of quantitation values of 1-50, 4-20 $\mu \mathrm{g} \mathrm{kg}^{-1}$ in full cream milk powders, skim milk powders, pure milks and acidophilus milks were calculated for PBDEs and MeO-PBDEs. In addition, good repeatability and accuracy of the whole method were achieved. Moreover, Eighteen kinds of commercial full cream milk powder samples, sixteen skim milk powder samples, twenty pure milk samples and fifteen acidophilus milk samples were collected from local supermarket in Wuhan, Hubei province. These samples were analyzed to determine whether these samples were contaminated by PBDEs and MeO-PBDEs. The method was successfully applied to determine methoxylated polybrominated diphenyl ethers and polybrominated diphenyl ethers in these dairy products. Using the established methods, PBDEs and MeO-PBDEs were not detected in all samples.
\end{abstract}

Keywords: MeO-PBDEs, PBDEs, Dairy Products, Isotopic Dilution, GC-MS

\section{Introduction}

Polybrominated diphenyl ethers (PBDEs) are one class of halogenated organic brominated flame retardants (BFRs), and have been used industrially in large volumes for flame protection purposes in various commercial products such as electronic equipment and textiles. The commercial PBDEs products predominantly consist of so-called penta-, octa- and decabromodiphenyl ether products. Because of their physical, chemical and bio-accumulative characteristics, such as environmental persistence and high lipophilicity, these products have been widely distributed in the environment, for example air, dust, fish and human milk, where they are found to persist for a long time [1-8].

Methoxylated polybrominated diphenyl ethers (MeO-PBDEs) are structural analogs to PBDEs. Firstly, to the best of our knowledge, MeO-PBDEs are not produced commercially. Secondly, some studies have revealed the presence of MeO-PBDEs in relatively high concentrations in marine environments, indicating a natural origin [9-11]. Thirdly, the presence of MeO-PBDEs in Arctic biota has been suggested to be caused by metabolic processes of precursor PBDEs, bioaccumulation of PBDE degradation products 
and/or naturally produced marine products [12]. A recent study also indicated that MeO-PBDEs occurred as metabolites of PBDEs [13]. In total, MeO-PBDEs have been considered anthropogenic compounds and reported as natural products present in the marine environment. In marine wildlife MeO-PBDEs were first analyzed in the aquatic Baltic environment, e.g., seal and fish from the Baltic Sea in year 1997 [14]. In recent years, MeO-PBDEs have been paid special attention mainly in biotic samples all over the world, and were found in eggs of white-tailed sea eagles breeding in different regions of Sweden [15], in fish and shellfish samples from the Mediterranean Sea [16], in mullet (Mugil cephalus) and sea bass (Dicentrarchus labrax) from Bizerte Lagoon, Tunisia [17], in mollusk and fish from the Bohai Sea and the Donghai Sea, China [18], in blue mussels from the Baltic Sea, Sweden [19], in Japanese common squid (Todarodes pacificus) from Korean offshore waters [20]. MeO-PBDEs are major contributors to the persistent organobromine load in sub-Arctic and Arctic marine mammals, covering a time period of more than 20 years [21].

As the PBDEs and MeO-PBDEs can be introduced into food chian by widespread environment pollution. Following concerns about contamination status of PBDEs and MeO-PBDEs in the dairy products, have led to the rising concern about the possible adverse health effects to humans. Toxicity studies indicate that the liver, thyroid gland and possibly also developing reproductive organs are particular targets of PBDEs toxicity [22, 23]. Evidence is emerging that PBDEs may be developmental neurotoxicants, as behavioural, neurochemical and hormonal deficits have been found following perinatal exposure [24-29]. PBDEs are capable to induce cell death of cerebellar granule cells in culture [30]. Madia et al reports PBDE-99 can induce apoptosis in astrocytoma cells assessed by the TUNEL method and by Hoechst 33258 staining, via a p53 dependent mechanism [31]. Our study indicates PBDE-209 can inhibit the proliferation of Hep G2 cells by inducing apoptosis through ROS generation [32]. A few researches about toxicity of MeO-PBDEs indicate the kind of compounds have effects on steroidogenic genes, aromatase activity and steroid hormones in vitro and may have the potential to affect steroidogenesis and reproduction in whole organisms [33, 34]. To satisfy the requirements of further accurate risk assessments for these chemicals, especially MeO-PBDEs, it is expected that the trend in generating MeO-PBDEs and PBDEs data will be encouraged to grow in dairy products samples.

Human exposure to PBDEs and MeO-PBDEs may occur via household dust, outdoor air, drinking water and food. PBDEs and MeO-PBDEs could be bio-accumulated in the food chain, in general, dietary intake was considered the most important route of human exposure. Dairy products have made and will always make important contributions to the human diet. They provide high quality protein and are good sources of vitamins and minerals, which satisfy the nutrient requirements of individuals. Therefore, it's necessary to quantify the content of PBDEs and MeO-PBDEs in dairy products. To our knowledge, there is little information about the degree of MeO-PBDEs and PBDEs contamination in dairy products in China. In addition, especially there are only few literatures about analysis method and contamination of PBDEs in human milk. The analysis of PBDEs and MeO-PBDEs in dairy products samples is difficult because they are usually present at $\mu \mathrm{g} \mathrm{kg}^{-1}$ levels and the matrices involved are generally complex. Beyond that, dairy products usually contain a large number of proteins, the proteins have stronger adsorption to the PBDEs. Therefore, highly selective and sensitive techniques including sample preparation, cleanup, instrument and quantitative method are required.

The present work describes a simultaneous determinations of fourteen PBDEs and eight MeO-PBDEs in dairy products by isotopic dilution GC-MS which is always more reliable. Four common dairy products were choosen as samples, including full cream milk powder, skim milk powder, pure milk and acidophilus milk. In addition, the contamination of MeO-PBDEs and PBDEs in these dairy products from the Hubei province of China was investigated.

\section{Experimental}

\subsection{Instrumentation and Reagents}

A 7890B-5977A GC-MS with electron impact ionization (EI) was purchased from Agilent (USA).

Acetone was supplied from JT Baker (Phillipsburg, USA). ethyl acetate, cyclohexane, iso-octane, were supplied from CNW (Germany). Activated silica gel column ( $5 \mathrm{~mm}$ i.d., Wako gel S-1) was from Wako Pure Chemical Industries Ltd., (Osaka, Japan). Gel permeation chromatography (GPC) (Bio-Beads S-X3) was supplied from Bio-Rad Laboratories (CA, USA). Florisil (60-100 mesh) was from Sigma-aldrich (USA).

A standard solution of native MeO-PBDEs containing the congeners 5-MeO-BDE47, 6-MeO-BDE47, 4'-MeO-BDE49, 2'-MeO-BDE68, 5'-MeO-BDE99, 5'-MeO-BDE100, 4'-MeO-BDE101 and 4'-MeO-BDE103, at a concentration of $5 \mu \mathrm{g} \mathrm{mL} L^{-1}$ in nonane and toluene (92:8) was supplied by Wellington Laboratories. A standard solution of native PBDEs containing congeners BDE17, BDE28, BDE47, BDE66, BDE71, BDE85, BDE99, BDE100, BDE138, BDE153, BDE154, BDE183 BDE190 and BDE209, at $5 \mu \mathrm{g} \mathrm{mL}$ of each congener in iso-octane and toluene (97.5:2.5), was also obtained from AccuStandard, Inc. (USA). For quantification by isotope dilution and internal standard, a standard mixture of ${ }^{13} \mathrm{C}_{12}$-labelled PBDEs 28, 47, 99, 100,154, 153 and 183 (MBDE-MXFS), supplied by Wellington Laboratories, at $2 \mu \mathrm{g}$ $\mathrm{mL}^{-1}$ of each congener in toluene and nonane (74.8:25.2) was used as surrogate internal standard. In addition, a standard mixture of ${ }^{13} \mathrm{C}_{12}$-BDE77 and 138 (MBDE-MXFR) at a concentration of $2 \mu \mathrm{g} \mathrm{mL}^{-1}$ in toluene and nonane (92.8:7.2) (Wellington Laboratories) was used as syringe standard for recovery determination. Mixed series working standards of MeO-PBDEs and PBDEs containing MBDE-MXFS and MBDE-MXFR were prepared by serial dilutions of these stock solutions with iso-octane. The detailed information of 
these standard substances was presented in Table 1.

\subsection{Dairy Products Samples}

Eighteen kinds of commercial full cream milk powder samples, sixteen skim milk powder samples, twenty pure milk samples and fifteen acidophilus milk samples were collected from local supermarket from Wuhan of Hubei province in March, 2015. All the samples were stored at $4{ }^{\circ} \mathrm{C}$ before extraction.

\subsection{Determination of MeO-PBDEs and PBDEs}

Analysis of MeO-PBDEs and PBDEs in dairy were prepared by using an literature method modified $[38,39]$. Accurately weighed samples $2 \mathrm{~g}$ ( liquid milk were freeze-dried ) and mixed with 10 volumes of Florisil spiked with IS ( $1 \mathrm{~mL}$ of the concentrations of ${ }^{13} \mathrm{C}_{12}$-labelled PBDEs $28,47,99,100,154,153$ and 183 were $100 \mathrm{ng} \mathrm{mL}-1)$. The mixtures were wetpacked with ethyl acetate/cyclohexane $(1: 1$, $\mathrm{v} / \mathrm{v})$ into a glass column $(25.0 \mathrm{~mm}$ i.d. $30.0 \mathrm{~cm})$. The the crude extracts were concentrated almost to dryness at $40^{\circ} \mathrm{C}$ in a water bath by a K-D vacuum rotary concentrator, and $2.0 \mathrm{~mL}$ of ethyl acetate/cyclohexane $(1: 1, \mathrm{v} / \mathrm{v})$ was added to dissolve the residue.

The resulting mixture was filtrated with a 0.22 um membrane and subjected to gel permeation chromatography (GPC) for lipid removal. The GPC column (30.0 mm i.d. 40.0 $\mathrm{cm})$ was packed with about $60 \mathrm{~g}$ (dry weight) of 200-400 mesh S-X3 Bio-Beads that had been soaked in ethyl acetate/cyclohexane mixture $(1: 1, \mathrm{v} / \mathrm{v})$ overnight, and the actual length of filling column was about $32.0 \mathrm{~cm}$. The column was conditioned with ethyl acetate/cyclohexane $(1: 1, \mathrm{v} / \mathrm{v})$, and the flow rate was $1.25 \mathrm{~mL} \mathrm{~min}^{-1}$. The first lipid fraction (F1, $100 \mathrm{~mL}$ ) from the GPC was discarded. The next fraction containing MeO-PBDEs and PBDEs (F2, $100 \mathrm{~mL}$ ) was concentrated almost to dryness at $40^{\circ} \mathrm{C}$ in a water bath by a $\mathrm{K}-\mathrm{D}$ vacuum rotary concentrator, and $5.0 \mathrm{~mL}$ of iso-octane was added to dissolve the residue. Then the residue was transferred to a glass tube, and concentrated almost to dryness by a gentle stream of nitrogen. $1 \mathrm{~mL}$ of iso-octane was added to dissolve the residues.

The residues were concentrated and further purified by passage over $1 \mathrm{~g}$ of activated silica gel column, with elution with $15 \mathrm{~mL}$ ethyl acetate/cyclohexane $(1: 1, \mathrm{v} / \mathrm{v})$. The eluate was reduced almost to dryness by a gentle stream of nitrogen. $0.95 \mathrm{~mL}$ of iso-octane followed by $0.05 \mathrm{~mL}$ syringe standard solution (the concentrations of ${ }^{13} \mathrm{C}_{12}$-BDE77 and 138 were 2 $\mu \mathrm{g} \mathrm{mL}^{-1}$ ) were added to dissolve the residue and transferred to an injection vial prior to GC-MS analysis. Sample blanks were taken through all aspects of the experimental procedure.

\subsection{GC-MS Conditions}

An Agilent 7890B-5977A GC-MS with electron impact ionization (EI) was used to the simultaneous determinations of MeO-PBDEs and PBDEs. An DB-5MS column ( $15 \mathrm{~m} \times 0.25$ $\mathrm{mm}$ (id), $0.25 \mu \mathrm{m}$ film thickness; Agilent, USA), was used to separate eight MeO-PBDEs, fourteen PBDEs, seven labeled
PBDEs (surrogate internal standard) and other two labeled PBDEs (syringe standard).

The oven temperature was initially set at $150^{\circ} \mathrm{C}$ for $1 \mathrm{~min}$, then increased to $325^{\circ} \mathrm{C}$ at a rate of $5^{\circ} \mathrm{C} \mathrm{min}^{-1}$, and held at $325^{\circ} \mathrm{C}$ for $5 \mathrm{~min}$. The total runtime was $41 \mathrm{~min}$. Helium was used as carrier gas at a constant flow rate of $1 \mathrm{~mL} \mathrm{~min}{ }^{-1} .4$ microliters of samples and standards were injected in splitless injection mode at an injector temperature of $325^{\circ} \mathrm{C}$.

MS operating conditions were the following: electron ionization mode using automatic gain control (AGC) with electron energy of $70 \mathrm{eV}$ and an emission current of $250 \mu \mathrm{A}$. The transfer line and ion source temperatures were kept at $320^{\circ} \mathrm{C}$ and $230^{\circ} \mathrm{C}$, respectively. The electron multiplier voltage was set to $370 \mathrm{~V}$. Filament delay was set $4 \mathrm{~min}$. The mass spectrometric date was acquired in SIM mode with selected ions for each analyt (Table 1).

\subsection{Quantification and Quality Control}

Our laboratory has established a quality assurance system as per ISO/IEC 17025: 2005 for strict controls over personnel, conditions of instruments, experimental situation, etc. Eight MeO-PBDEs and fourteen PBDEs were quantified by isotope dilution or internal standard using MBDE-MXFS, and the quantification of real samples was dealt with by isotope dilution or internal standard in this study. On the one hand, isotope dilution was used for calibration for MeO-PBDEs and PBDEs that have a labeled analog, for example, for BDE28, on the other hand, internal standard was applied to determination of MeO-PBDEs and PBDEs for which a labeled analog was not used in the study. The detailed information of quantitation reference for eight $\mathrm{MeO}-\mathrm{PBDEs}$ and fourteen PBDEs, was given in Table 2.

In order to ensure the accuracy of the results and the applicability of the method in the study, in the case of repeated analyses of the spiking and real samples, the guideline ranges for the deviation of the experimentally determined recovery corrected mean mass fraction from the spiking value must meet the requirements of the European Union document 2002/657/EC: Commission Decision of 12 August 2002 implementing Council Directive 96/23/EC concerning the performance of analytical methods and the interpretation of results. In addition, the recovery efficiency of all MBDE-MXFS by MBDE-MXFR shall be $60 \%$ or better.

\section{Results}

\subsection{GC-MS Parameters for MeO-PBDEs and PBDEs}

According to GC-MS conditions in the second section, the information of retention times (RT), start time and end time of retention window, quantitation reference for MeO-PBDEs, PBDEs, MBDE-MXFS and MBDE-MXFR on DB-5MS were presented in Table 2.

Quantitative determination by GC-MS (EI+) was in the selected ion monitoring (SIM) mode. The ions monitored for PBDEs and MeO-PBDEs, including labeled PBDEs were presented in Table 1. 
Table 1. The information and the ions monitored of fourteen PBDEs, eight MeO-PBDEs, MBDE-MXFS and MBDE-MXFR.

\begin{tabular}{|c|c|c|c|c|c|}
\hline Br No. & Abbreviation & Molar mass & $\begin{array}{l}\text { Precursor ions, }[\mathrm{M}]^{+} \text {and } \\
{[\mathrm{M}+2]^{+}, \text {or }[\mathrm{M}-2]^{+}(\mathrm{m} / \mathrm{z})}\end{array}$ & $\begin{array}{l}\text { Product ions, }[\mathrm{M}-2 \mathrm{Br}]^{+} \text {and } \\
{[(\mathrm{M}+2)-2 \mathrm{Br}]^{+}, \text {or }[(\mathrm{M}-2)-2 \mathrm{Br}]^{+}(\mathrm{m} / \mathrm{z})}\end{array}$ & $\begin{array}{l}\text { The ions monitored } \\
(\mathrm{m} / \mathbf{z})\end{array}$ \\
\hline \multicolumn{6}{|l|}{ PBDEs } \\
\hline 3 & BDE17 & 407 & $406,[\mathrm{M}+2]^{+}$ & 246,248 & $406,408, \underline{246}, 248$ \\
\hline 4 & BDE71 & 486 & $486,[\mathrm{M}-2]^{+}$ & 326,328 & $484,486, \underline{326}, 328$ \\
\hline 4 & BDE47 & 486 & $486,[\mathrm{M}-2]^{+}$ & 326,328 & $484,486, \underline{326}, 328$ \\
\hline 4 & BDE66 & 486 & $486,[\mathrm{M}-2]^{+}$ & 326,328 & $484,486, \underline{326}, 328$ \\
\hline 5 & BDE99 & 565 & $564,[\mathrm{M}+2]^{+}$ & 404,406 & $564,566, \underline{404}, 406$ \\
\hline 5 & BDE85 & 565 & $564,[\mathrm{M}+2]^{+}$ & 404,406 & $564,566, \underline{404}, 406$ \\
\hline 6 & BDE154 & 644 & $644,[\mathrm{M}-2]^{+}$ & 484,486 & $642,644, \underline{484}, 486$ \\
\hline 6 & BDE153 & 644 & $644,[\mathrm{M}-2]^{+}$ & 484,486 & $642,644,484,486$ \\
\hline 6 & BDE138 & 644 & $644,[\mathrm{M}-2]^{+}$ & 484,486 & $642,644, \underline{484}, 486$ \\
\hline 7 & BDE183 & 723 & $722,[\mathrm{M}+2]^{+}$ & 562,564 & $722,724,562, \underline{564}$ \\
\hline 4 & 6-MeO-BDE47 & 516 & $516,[\mathrm{M}-2]^{+}$ & $356,420,422,{ }^{*}\left[\mathrm{M}-\mathrm{CH}_{3} \mathrm{Br}\right]^{+}$ & $514, \underline{516}, 420,422,356$ \\
\hline 4 & 5-MeO-BDE47 & 516 & $516,[\mathrm{M}-2]^{+}$ & 356,358 & $514,516,356,358$ \\
\hline 4 & 4'-MeO-BDE49 & 516 & $516,[\mathrm{M}-2]^{+}$ & 356,358 & $514, \underline{516}, 356,358$ \\
\hline 5 & 5'-MeO-BDE100 & 595 & $596,[\mathrm{M}-2]^{+}$ & 434,436 & $594,596,434,436$ \\
\hline 5 & 4'-MeO-BDE103 & 595 & $596,[\mathrm{M}-2]^{+}$ & 434,436 & $\underline{594,596,434,436}$ \\
\hline 5 & 5'-MeO-BDE99 & 595 & $596,[\mathrm{M}-2]^{+}$ & 434,436 & $\overline{594,596,434,436}$ \\
\hline 5 & 4'-MeO-BDE101 & 595 & $596,[\mathrm{M}-2]^{+}$ & 434,436 & $\underline{594,596,434,436}$ \\
\hline \multicolumn{6}{|c|}{ MBDE-MXFS } \\
\hline 3 & ${ }^{13} \mathrm{C}_{12}$-BDE-28 & 419 & $418,[\mathrm{M}+2]^{+}$ & 258,260 & $418,420, \underline{258}, 260$ \\
\hline 4 & ${ }^{13} \mathrm{C}_{12}-\mathrm{BDE}-47$ & 500 & $498,[\mathrm{M}+2]^{+}$ & 338,340 & $498,500, \underline{338}, 340$ \\
\hline 5 & ${ }^{13} \mathrm{C}_{12}$-BDE-100 & 577 & $576,[\mathrm{M}+2]^{+}$ & 416,418 & $576,578, \underline{416}, 418$ \\
\hline
\end{tabular}

The ion of underline was indicated for quantitative analysis.

Table 2. Retention times (RT), start time and end time of retention window, quantitation reference for MeO-PBDEs, PBDEs, MBDE-MXFS and MBDE-MXFR on $D B-5 M S$, and $L O Q$ of MeO-PBDEs and PBDEs in full cream milk powders, skim milk powders, pure milks and acidophilus milks.

\begin{tabular}{|c|c|c|c|c|c|}
\hline \multirow[b]{2}{*}{ Compounds } & \multirow[b]{2}{*}{$\begin{array}{l}\text { Quantitation } \\
\text { reference }\end{array}$} & \multirow[b]{2}{*}{$\begin{array}{l}\text { Retention } \\
\text { times (RT) }\end{array}$} & \multirow{2}{*}{$\begin{array}{l}\text { Start time and end } \\
\text { time of Retention } \\
\text { Window }\end{array}$} & \multicolumn{2}{|l|}{ LOQ } \\
\hline & & & & $\begin{array}{l}\text { full cream milk powders and } \\
\text { skim milk powders }\left(\mu \mathrm{gg}^{-1}\right)\end{array}$ & $\begin{array}{l}\text { pure milks and acidophilus } \\
\text { milks }\left(\mu \mathrm{gg}^{-1}\right)\end{array}$ \\
\hline \multicolumn{6}{|c|}{ Compounds using ${ }^{13} \mathrm{C}_{12}$-BDE-77 as Labeled injection internal standard } \\
\hline BDE28 & ${ }^{13} \mathrm{C}_{12}$-BDE-28 & 12.18 & $12 \sim 13$ & 1 & 1 \\
\hline BDE71 & ${ }^{13} \mathrm{C}_{12}-\mathrm{BDE}-47$ & 15.66 & $13 \sim 16$ & 2 & 2 \\
\hline BDE47 & ${ }^{13} \mathrm{C}_{12}$-BDE-47 & 16.15 & $16 \sim 16.5$ & 2 & 2 \\
\hline BDE100 & ${ }^{13} \mathrm{C}_{12}$-BDE-100 & 19.05 & $18.6 \sim 19.15$ & 4 & 4 \\
\hline BDE99 & ${ }^{13} \mathrm{C}_{12}$-BDE-99 & 19.95 & $19.8 \sim 20.2$ & 4 & 4 \\
\hline BDE85 & ${ }^{13} \mathrm{C}_{12}$-BDE-99 & 21.44 & $21.4 \sim 22.1$ & 4 & 4 \\
\hline \multicolumn{6}{|c|}{ Compounds using ${ }^{13} \mathrm{C}_{12}-\mathrm{BDE}-138$ as Labeled injection internal standard } \\
\hline BDE154 & ${ }^{13} \mathrm{C}_{12}$-BDE-154 & 22.19 & $22.1 \sim 22.5$ & 4 & 4 \\
\hline BDE153 & ${ }^{13} \mathrm{C}_{12}-\mathrm{BDE}-153$ & 23.41 & $23.2 \sim 24.7$ & 4 & 4 \\
\hline
\end{tabular}




\begin{tabular}{|c|c|c|c|c|c|}
\hline \multirow[b]{2}{*}{ Compounds } & \multirow{2}{*}{$\begin{array}{l}\text { Quantitation } \\
\text { reference }\end{array}$} & \multirow{2}{*}{$\begin{array}{l}\text { Retention } \\
\text { times (RT) }\end{array}$} & \multirow{2}{*}{$\begin{array}{l}\text { Start time and end } \\
\text { time of Retention } \\
\text { Window }\end{array}$} & \multicolumn{2}{|l|}{ LOQ } \\
\hline & & & & $\begin{array}{l}\text { full cream milk powders and } \\
\text { skim milk powders }\left(\mu \mathrm{g} \mathrm{kg}^{-1}\right)\end{array}$ & $\begin{array}{l}\text { pure milks and acidophilus } \\
\text { milks }\left(\mu \mathrm{g} \mathrm{kg}^{-1}\right)\end{array}$ \\
\hline BDE183 & ${ }^{13} \mathrm{C}_{12}-\mathrm{BDE}-183$ & 26.57 & $26.0 \sim 28.0$ & 10 & 10 \\
\hline BDE209 & ${ }^{13} \mathrm{C}_{12}$-BDE-183 & 38.35 & $30.0 \sim 41$ & 50 & 50 \\
\hline \multicolumn{6}{|c|}{ Compounds using ${ }^{13} \mathrm{C}_{12}$-BDE-77 as Labeled injection internal standard } \\
\hline 2'-MeO-BDE68 & ${ }^{13} \mathrm{C}_{12}$-BDE-100 & 17.70 & $17.0 \sim 18.1$ & 4 & 4 \\
\hline 4'-MeO-BDE49 & ${ }^{13} \mathrm{C}_{12}$-BDE-100 & 19.42 & $19.4 \sim 19.8$ & 10 & 10 \\
\hline \multicolumn{6}{|c|}{ Compounds using ${ }^{13} \mathrm{C}_{12}-\mathrm{BDE}-138$ as Labeled injection internal standard } \\
\hline 5'-MeO-BDE100 & ${ }^{13} \mathrm{C}_{12}$-BDE-154 & 21.17 & $20.2 \sim 21.4$ & 10 & 10 \\
\hline 4'-MeO-BDE103 & ${ }^{13} \mathrm{C}_{12}-\mathrm{BDE}-154$ & 21.44 & $21.4 \sim 22.1$ & 10 & 10 \\
\hline 5'-MeO-BDE99 & ${ }^{13} \mathrm{C}_{12}$-BDE-154 & 22.50 & $22.5 \sim 22.81$ & 10 & 10 \\
\hline${ }^{13} \mathrm{C}_{12}$-BDE-100 & ${ }^{13} \mathrm{C}_{12}-\mathrm{BDE}-77$ & 19.05 & $18.6 \sim 19.15$ & - & - \\
\hline${ }^{13} \mathrm{C}_{12}$-BDE-99 & ${ }^{13} \mathrm{C}_{12}-\mathrm{BDE}-77$ & 19.95 & $19.8 \sim 20.2$ & - & - \\
\hline${ }^{13} \mathrm{C}_{12}-\mathrm{BDE}-154$ & ${ }^{13} \mathrm{C}_{12}-\mathrm{BDE}-138$ & 22.19 & $22.1 \sim 22.5$ & - & - \\
\hline${ }^{13} \mathrm{C}_{12}$-BDE-153 & ${ }^{13} \mathrm{C}_{12}$-BDE-138 & 23.41 & $23.2 \sim 24.7$ & - & - \\
\hline${ }^{13} \mathrm{C}_{12}$-BDE-183 & ${ }^{13} \mathrm{C}_{12}$-BDE-138 & 26.57 & $26.0 \sim 28.0$ & - & - \\
\hline \multicolumn{6}{|c|}{ Labeled injection internal standards } \\
\hline${ }^{13} \mathrm{C}_{12}-\mathrm{BDE}-77$ & ${ }^{13} \mathrm{C}_{12}$-BDE-77 & 17.73 & $17.0 \sim 18.1$ & - & - \\
\hline${ }^{13} \mathrm{C}_{12}$-BDE-138 & ${ }^{13} \mathrm{C}_{12}$-BDE-138 & 24.91 & $24.7 \sim 26.0$ & - & - \\
\hline
\end{tabular}

The transverse line was indicated no data.

Table 3. Seven mixed series working standards of $M e O-P B D E s, P B D E s, M B D E-M X F S$ and $M B D E-M X F R$.

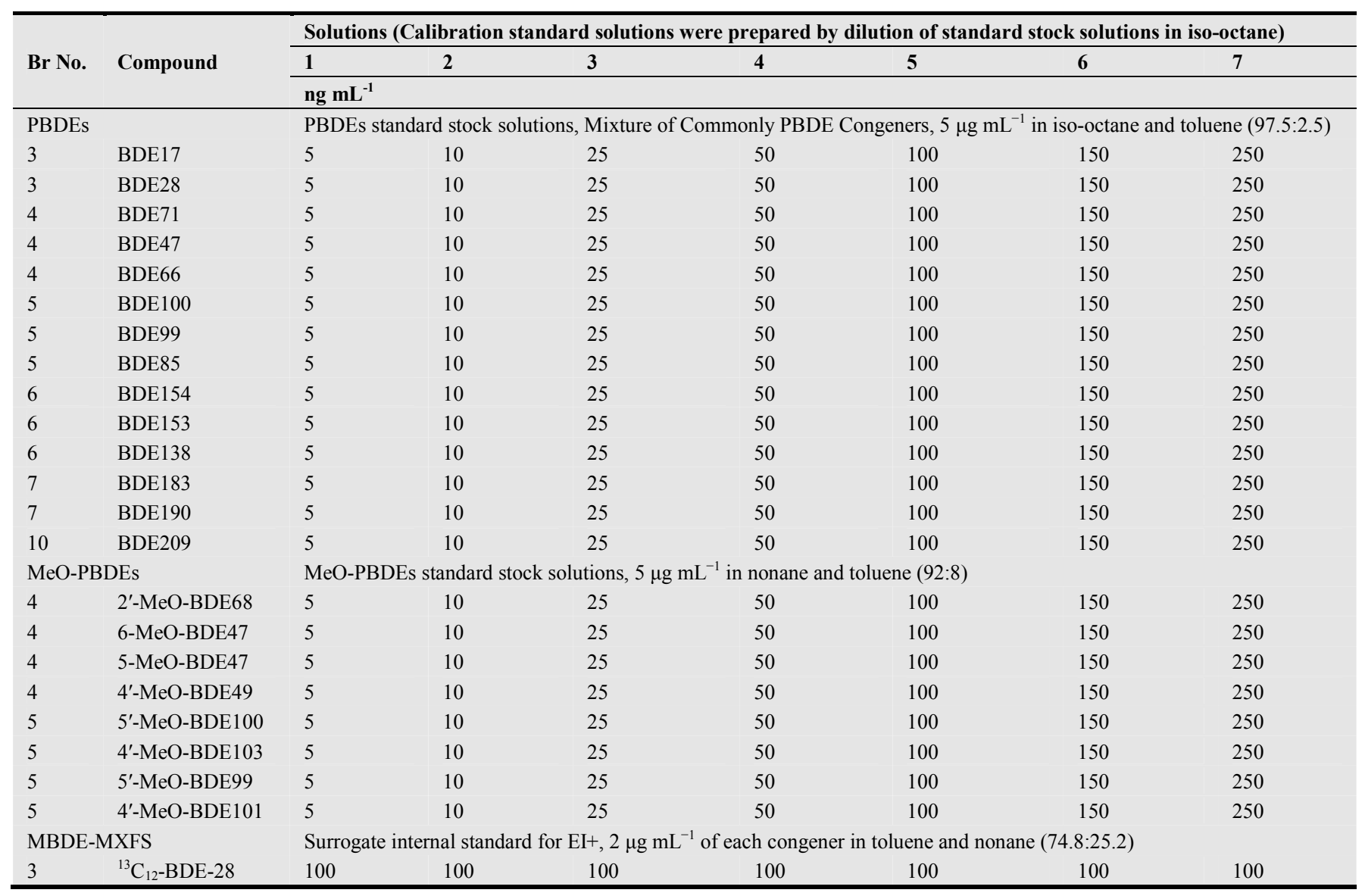




\begin{tabular}{|c|c|c|c|c|c|c|c|c|}
\hline \multirow{3}{*}{ Br No. } & \multirow{3}{*}{ Compound } & \multicolumn{7}{|c|}{ Solutions (Calibration standard solutions were prepared by dilution of standard stock solutions in iso-octane) } \\
\hline & & 1 & 2 & 3 & 4 & 5 & 6 & 7 \\
\hline & & \multicolumn{7}{|c|}{ ng mL $\mathbf{~ L ~}^{-1}$} \\
\hline 4 & ${ }^{13} \mathrm{C}_{12}-\mathrm{BDE}-47$ & 100 & 100 & 100 & 100 & 100 & 100 & 100 \\
\hline 5 & ${ }^{13} \mathrm{C}_{12}$-BDE-99 & 100 & 100 & 100 & 100 & 100 & 100 & 100 \\
\hline 6 & ${ }^{13} \mathrm{C}_{12}$-BDE-154 & 100 & 100 & 100 & 100 & 100 & 100 & 100 \\
\hline \multicolumn{2}{|c|}{ MBDE-MXFR } & \multicolumn{7}{|c|}{ Syringe standards, $2 \mu \mathrm{g} \mathrm{mL}^{-1}$ in toluene and nonane (92.8:7.2) } \\
\hline 4 & ${ }^{13} \mathrm{C}_{12}-\mathrm{BDE}-77$ & 100 & 100 & 100 & 100 & 100 & 100 & 100 \\
\hline 6 & ${ }^{13} \mathrm{C}_{12}$-BDE-138 & 100 & 100 & 100 & 100 & 100 & 100 & 100 \\
\hline
\end{tabular}

Table 4. Average recoveries and standard deviation of MeO-PBDEs and PBDEs in full cream milk powders, skim milk powders, pure milks and acidophilus milks $(\%$ $\pm S D, n=6)\left(\mu g \mathrm{~kg}^{-1}\right.$ dry weight).

\begin{tabular}{|c|c|c|c|c|c|c|c|c|c|c|c|c|c|c|}
\hline \multirow{2}{*}{$\begin{array}{l}\text { Br } \\
\text { No. }\end{array}$} & \multirow[t]{2}{*}{ Compound } & \multirow{2}{*}{$\begin{array}{l}\text { Quantitation } \\
\text { reference }\end{array}$} & \multicolumn{3}{|c|}{$\begin{array}{l}\text { Recoveries from full } \\
\text { cream milk powders }\end{array}$} & \multicolumn{3}{|c|}{$\begin{array}{l}\text { Recoveries from skim } \\
\text { milk powders }\end{array}$} & \multicolumn{3}{|c|}{$\begin{array}{l}\text { Recoveries from pure } \\
\text { milks }\end{array}$} & \multicolumn{3}{|c|}{$\begin{array}{l}\text { Recoveries from } \\
\text { acidophilus milks }\end{array}$} \\
\hline & & & 2.5 & 10 & 25 & 2.5 & 10 & 25 & 2.5 & 10 & 25 & 2.5 & 10 & 25 \\
\hline \multicolumn{15}{|c|}{ Compounds using ${ }^{13} \mathrm{C}_{12}$-BDE-77 as Labeled injection internal standard } \\
\hline 3 & BDE17 & ${ }^{13} \mathrm{C}_{12}-\mathrm{BDE}-28$ & $81 \pm 7$ & $93 \pm 5$ & $95 \pm 6$ & $71 \pm 10$ & $83 \pm 8$ & $88 \pm 12$ & $88 \pm 5$ & $91 \pm 5$ & $102 \pm 6$ & $91 \pm 13$ & $99 \pm 8$ & $103 \pm 7$ \\
\hline 3 & BDE28 & ${ }^{13} \mathrm{C}_{12}-\mathrm{BDE}-28$ & $86 \pm 7$ & $95 \pm 5$ & $92 \pm 8$ & $89 \pm 13$ & $92 \pm 7$ & $91 \pm 8$ & $72 \pm 6$ & $98 \pm 7$ & $97 \pm 9$ & $72 \pm 6$ & $100 \pm 8$ & $101 \pm 4$ \\
\hline 4 & BDE71 & ${ }^{13} \mathrm{C}_{12}$-BDE-47 & $75 \pm 4$ & $88 \pm 10$ & $92 \pm 5$ & $75 \pm 11$ & $90 \pm 11$ & $91 \pm 10$ & $77 \pm 6$ & $101 \pm 9$ & $101 \pm 7$ & $88 \pm 11$ & $98 \pm 7$ & $96 \pm 6$ \\
\hline 4 & BDE47 & ${ }^{13} \mathrm{C}_{12}$-BDE-47 & $87 \pm 5$ & $85 \pm 5$ & $92 \pm 7$ & $84 \pm 5$ & $82 \pm 8$ & $91 \pm 10$ & $87 \pm 7$ & $99 \pm 6$ & $101 \pm 7$ & $81 \pm 7$ & $100 \pm 5$ & $101 \pm 2$ \\
\hline 4 & BDE66 & ${ }^{13} \mathrm{C}_{12}$-BDE-47 & $78 \pm 9$ & $86 \pm 5$ & $93 \pm 12$ & $75 \pm 5$ & $90 \pm 10$ & $90 \pm 10$ & $84 \pm 9$ & $101 \pm 9$ & $95 \pm 9$ & $88 \pm 10$ & $95 \pm 6$ & $97 \pm 5$ \\
\hline 5 & BDE100 & ${ }^{13} \mathrm{C}_{12}$-BDE-100 & $81 \pm 13$ & $83 \pm 5$ & $95 \pm 5$ & $91 \pm 13$ & $86 \pm 9$ & $96 \pm 4$ & $89 \pm 8$ & $103 \pm 8$ & $103 \pm 8$ & $87 \pm 8$ & $99 \pm 8$ & $98 \pm 6$ \\
\hline 5 & BDE99 & ${ }^{13} \mathrm{C}_{12}$-BDE-99 & $81 \pm 14$ & $85 \pm 7$ & $88 \pm 9$ & $81 \pm 10$ & $86 \pm 7$ & $93 \pm 10$ & $90 \pm 12$ & $96 \pm 7$ & $97 \pm 5$ & $93 \pm 10$ & $93 \pm 10$ & $102 \pm 5$ \\
\hline 5 & BDE85 & ${ }^{13} \mathrm{C}_{12}$-BDE-99 & $78 \pm 16$ & $85 \pm 6$ & $82 \pm 6$ & $78 \pm 5$ & $91 \pm 9$ & $92 \pm 8$ & $87 \pm 5$ & $93 \pm 6$ & $104 \pm 5$ & $86 \pm 7$ & $86 \pm 5$ & $97 \pm 7$ \\
\hline \multicolumn{15}{|c|}{ Compounds using ${ }^{13} \mathrm{C}_{12}-\mathrm{BDE}-138$ as Labeled injection internal standard } \\
\hline 6 & BDE154 & ${ }^{13} \mathrm{C}_{12}-\mathrm{BDE}-154$ & $74 \pm 5$ & $82 \pm 6$ & $85 \pm 7$ & $80 \pm 6$ & $85 \pm 8$ & $88 \pm 12$ & $87 \pm 9$ & $96 \pm 8$ & $96 \pm 9$ & $86 \pm 4$ & $90 \pm 9$ & $101 \pm 7$ \\
\hline 6 & BDE153 & ${ }^{13} \mathrm{C}_{12}$-BDE-153 & $75 \pm 7$ & $82 \pm 4$ & $87 \pm 8$ & $73 \pm 7$ & $82 \pm 5$ & $93 \pm 6$ & $88 \pm 9$ & $90 \pm 7$ & $92 \pm 4$ & $82 \pm 8$ & $89 \pm 7$ & $98 \pm 8$ \\
\hline 6 & BDE138 & ${ }^{13} \mathrm{C}_{12}$-BDE-153 & $72 \pm 8$ & $81 \pm 6$ & $90 \pm 7$ & $71 \pm 6$ & $85 \pm 10$ & $82 \pm 4$ & $85 \pm 7$ & $87 \pm 6$ & $94 \pm 7$ & $83 \pm 11$ & $90 \pm 9$ & $90 \pm 10$ \\
\hline 7 & BDE183 & ${ }^{13} \mathrm{C}_{12}$-BDE-183 & $83 \pm 8$ & $83 \pm 3$ & $85 \pm 5$ & $78 \pm 9$ & $87 \pm 12$ & $85 \pm 4$ & $85 \pm 4$ & $94 \pm 11$ & $91 \pm 8$ & $75 \pm 8$ & $91 \pm 10$ & $91 \pm 6$ \\
\hline 7 & BDE190 & ${ }^{13} \mathrm{C}_{12}$-BDE- 183 & - & $80 \pm 14$ & $84 \pm 5$ & - & $82 \pm 14$ & $84 \pm 8$ & - & $91 \pm 9$ & $90 \pm 5$ & - & $88 \pm 9$ & $87 \pm 6$ \\
\hline \multicolumn{15}{|c|}{ Compounds using ${ }^{13} \mathrm{C}_{12}$-BDE-77 as Labeled injection internal standard } \\
\hline 4 & 2'-MeO-BDE68 & ${ }^{13} \mathrm{C}_{12}$-BDE-100 & $82 \pm 12$ & $92 \pm 7$ & $102 \pm 10$ & $77 \pm 9$ & $92 \pm 3$ & $96 \pm 12$ & $82 \pm 6$ & $103 \pm 6$ & $102 \pm 6$ & $100 \pm 9$ & $102 \pm 8$ & $102 \pm 6$ \\
\hline 4 & 6-MeO-BDE47 & ${ }^{13} \mathrm{C}_{12}$-BDE-100 & $76 \pm 7$ & $93 \pm 13$ & $96 \pm 8$ & $75 \pm 8$ & $100 \pm 6$ & $100 \pm 9$ & $100 \pm 6$ & $98 \pm 8$ & $98 \pm 8$ & $103 \pm 7$ & $103 \pm 9$ & $103 \pm 7$ \\
\hline 4 & 5-MeO-BDE47 & ${ }^{13} \mathrm{C}_{12}$-BDE-100 & $78 \pm 8$ & $99 \pm 8$ & $97 \pm 4$ & $75 \pm 5$ & $92 \pm 10$ & $94 \pm 12$ & $98 \pm 9$ & $99 \pm 8$ & $101 \pm 9$ & $92 \pm 11$ & $98 \pm 7$ & $100 \pm 6$ \\
\hline 4 & $4^{\prime}-\mathrm{MeO}-\mathrm{BDE} 49$ & ${ }^{13} \mathrm{C}_{12}-\mathrm{BDE}-100$ & $79 \pm 8$ & $86 \pm 9$ & $91 \pm 6$ & $89 \pm 4$ & $95 \pm 8$ & $95 \pm 7$ & $95 \pm 7$ & $92 \pm 6$ & $100 \pm 4$ & $93 \pm 7$ & $94 \pm 7$ & $100 \pm 5$ \\
\hline \multicolumn{15}{|c|}{ Compounds using ${ }^{13} \mathrm{C}_{12}$-BDE-138 as Labeled injection internal standard } \\
\hline 5 & 5'-MeO-BDE100 & ${ }^{13} \mathrm{C}_{12}$-BDE- 154 & $79 \pm 12$ & $86 \pm 6$ & $91 \pm 4$ & $76 \pm 11$ & $86 \pm 5$ & $85 \pm 5$ & $95 \pm 4$ & $97 \pm 5$ & $102 \pm 8$ & $88 \pm 8$ & $99 \pm 7$ & $100 \pm 7$ \\
\hline 5 & 4'-MeO-BDE103 & ${ }^{13} \mathrm{C}_{12}$-BDE-154 & $78 \pm 7$ & $85 \pm 4$ & $87 \pm 4$ & $72 \pm 6$ & $87 \pm 10$ & $89 \pm 10$ & $86 \pm 5$ & $97 \pm 6$ & $100 \pm 7$ & $89 \pm 12$ & $104 \pm 7$ & $98 \pm 12$ \\
\hline 5 & 5'-MeO-BDE99 & ${ }^{13} \mathrm{C}_{12}$-BDE-154 & $80 \pm 7$ & $84 \pm 7$ & $88 \pm 5$ & $80 \pm 13$ & $85 \pm 10$ & $89 \pm 6$ & $88 \pm 10$ & $101 \pm 7$ & $100 \pm 6$ & $86 \pm 8$ & $94 \pm 6$ & $103 \pm 5$ \\
\hline 5 & 4'-MeO-BDE101 & ${ }^{13} \mathrm{C}_{12}$-BDE-154 & - & $82 \pm 12$ & $88 \pm 5$ & - & $81 \pm 8$ & $87 \pm 5$ & - & $97 \pm 7$ & $95 \pm 6$ & - & $92 \pm 6$ & $94 \pm 4$ \\
\hline
\end{tabular}

The transverse line was indicated no data because the spiked amount was below LOQ.

\subsection{Calibration Curves, Limits of Quantitation and Recovery Rates}

Seven mixed series working standards of MeO-PBDEs and PBDEs, including MBDE-MXFS and MBDE-MXFR were prepared according to Table 3. Linear calibration curves for MeO-PBDEs and PBDEs by isotopic dilution and internal standard method were obtained with a $\gamma 2$ correlation coefficient of more than 0.99 . The linearity was checked by calculating the standard deviation of the average of response factors (peak area ratios divided by the corresponding analyte concentration ratios of all standards), which was $<15 \%$ assuming a linear response.

The limit of quantitation (LOQ), defined as the concentration of analyte which yielded a peak-to-peak signal-to-noise ratio of at least 10:1, was calculated by running a series of 10 negative extracts. The detailed information for LOQ of full cream milk powder, skim milk powder, pure milk and acidophilus milk was presented in Table 2.

Spike recoveries of MeO-PBDEs and PBDEs in these productions were studied. Table 4 provided further information. Average recoveries and standard deviation (SD) of all concentrations analyzed triple samples met the requirements of the European Union document 2002/657/EC: Commission Decision of 12 August 2002 implementing Council Directive 96/23/EC concerning the performance of analytical methods and the interpretation of results. In addition, the recovery efficiency of all MBDE-MXFS by 
MBDE-MXFR was more than $80 \%$.

\subsection{Analysis of Dairy Products}

GC-MS chromatogram of a mixed standard solution of MeO-PBDEs and PBDEs (the concentration of BDE17, BDE28, BDE47, BDE66, BDE71, BDE85, BDE99, BDE100, BDE138, BDE153, BDE154, BDE183 BDE190, and BDE209 were $100 \mathrm{ng} \mathrm{mL}^{-1}$, respectively, the concentration of 5-MeO-BDE47, 6-MeO-BDE47, 4'-MeO-BDE49, 2'-MeO-BDE68, 5'-MeO-BDE99, 5'-MeO-BDE100, $4^{\prime}-\mathrm{MeO}-\mathrm{BDE} 101$ and $4^{\prime}-\mathrm{MeO}-\mathrm{BDE} 103$ were $100 \mathrm{ng} \mathrm{mL}{ }^{-1}$, respectively, the concentrations of ${ }^{13} \mathrm{C}_{12}$-labelled PBDEs 28, $47,99,100,154,153$ and 183 as surrogate internal standard were $100 \mathrm{ng} \mathrm{mL}$, respectively, and the concentration of
${ }^{13} \mathrm{C}_{12}$-labelled PBDEs 77 and 138 as syringe standard were $100 \mathrm{ng} \mathrm{mL}^{-1}$, respectively), was shown in Figure 1. Because PBDEs, MeO-PBDEs and isotope internal standards have similar structure and property, it causes some of those substances have same chromatographic behavior, which is difficult to separate.

Using the established methods, 18 full cream milk powder samples, 16 skim milk powder samples, 20 pure milk samples and 15 acidophilus milk samples were tested. MeO-PBDEs or PBDEs were not found in all dairy products. GC-MS chromatogram of the spiked pure milk sample (the spiked level was $100 \mu \mathrm{g} \mathrm{kg}^{-1}$ ) was given in Figure 2. GC-MS chromatogram of the blank pure milk sample was given in Figure 3.

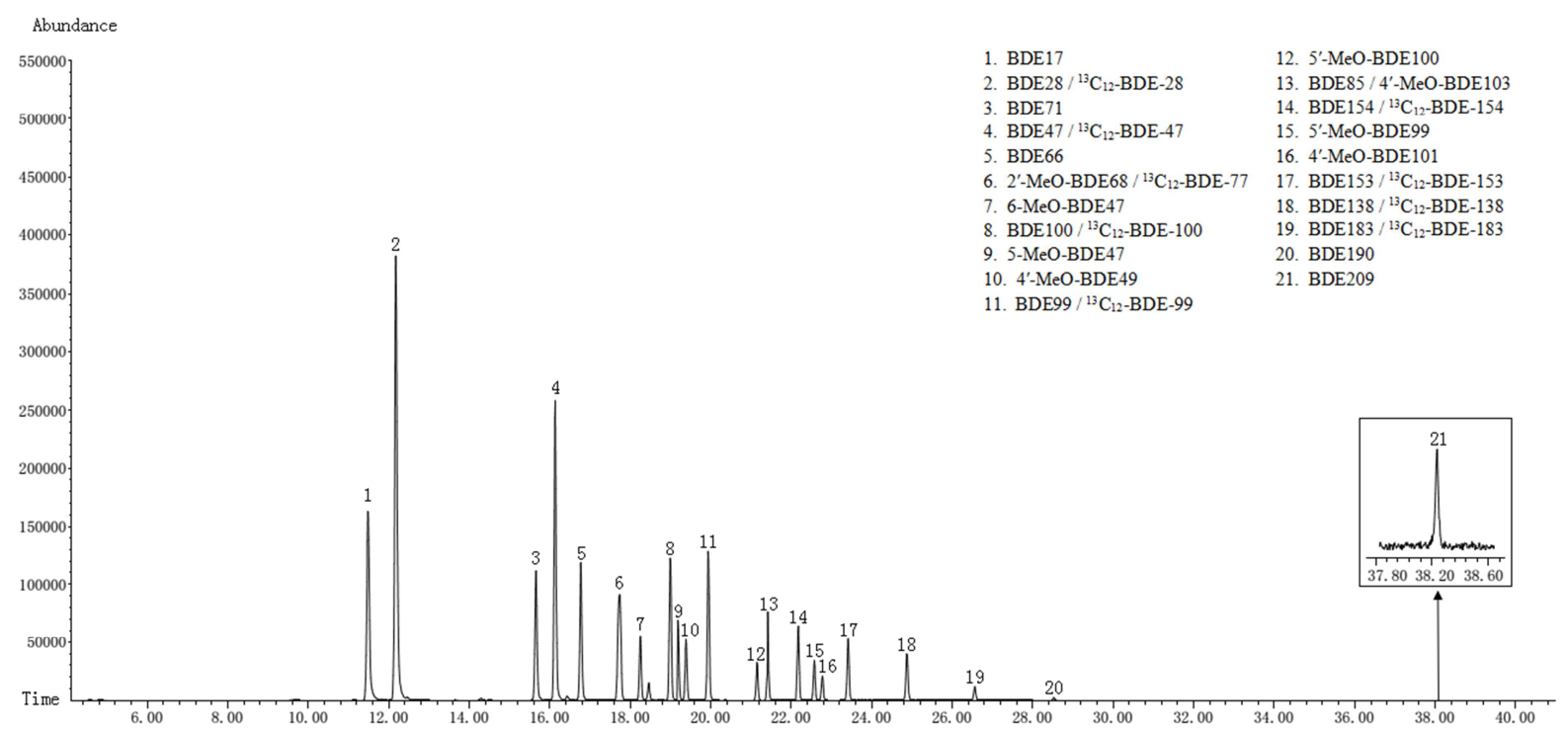

Figure 1. GC-MS chromatogram of a mixed standard solution of MeO-PBDEs and PBDEs (the concentration of BDE17, BDE28, BDE47, BDE66, BDE71, BDE85, BDE99, BDE100, BDE138, BDE153, BDE154, BDE183, BDE190 and BDE209 were $100 \mathrm{ng}^{\mathrm{mL}} \mathrm{H}^{-1}$, respectively, the concentration of 5-MeO-BDE47, 6-MeO-BDE47, 4'-MeO-BDE49, 2'-MeO-BDE68, 5'-MeO-BDE99, 5'-MeO-BDE100, 4'-MeO-BDE101 and 4'-MeO-BDE103 were 100 ng mL-1, respectively, the concentrations of ${ }^{13} C_{12}$-labelled PBDEs 28, 47, 99, 100, 154, 153 and 183 were $100 \mathrm{ng} \mathrm{mL}^{-1}$, respectively, and the concentration of ${ }^{13} C_{12}$-labelled PBDEs 77 and 138 were $100 \mathrm{ng}^{-1}$, respectively).

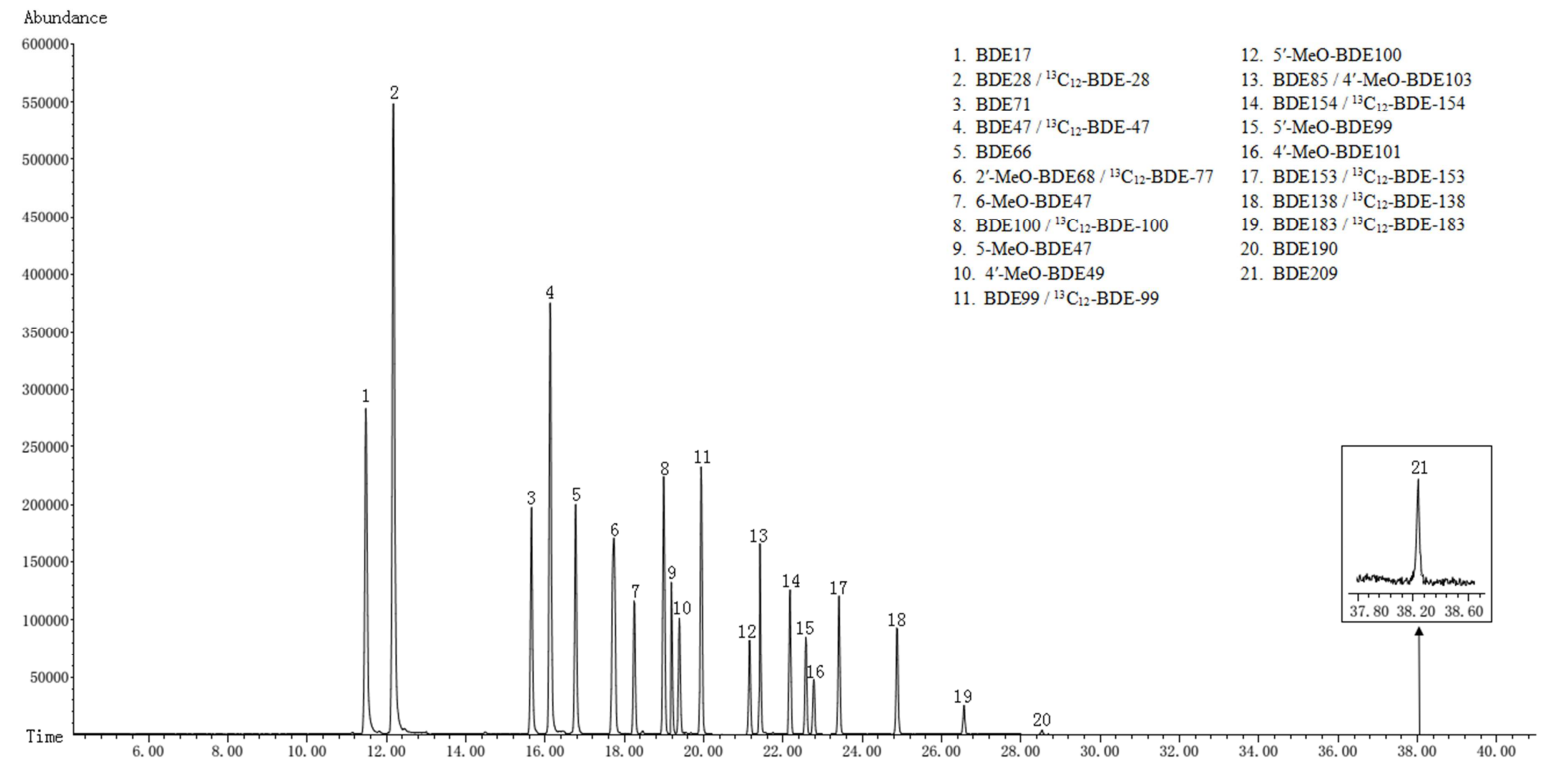

Figure 2. GC-MS chromatogram of a blank pure milk extract fortified with MeO-PBDEs and PBDEs at $100 \mu \mathrm{g} \mathrm{kg}^{-1}$. 


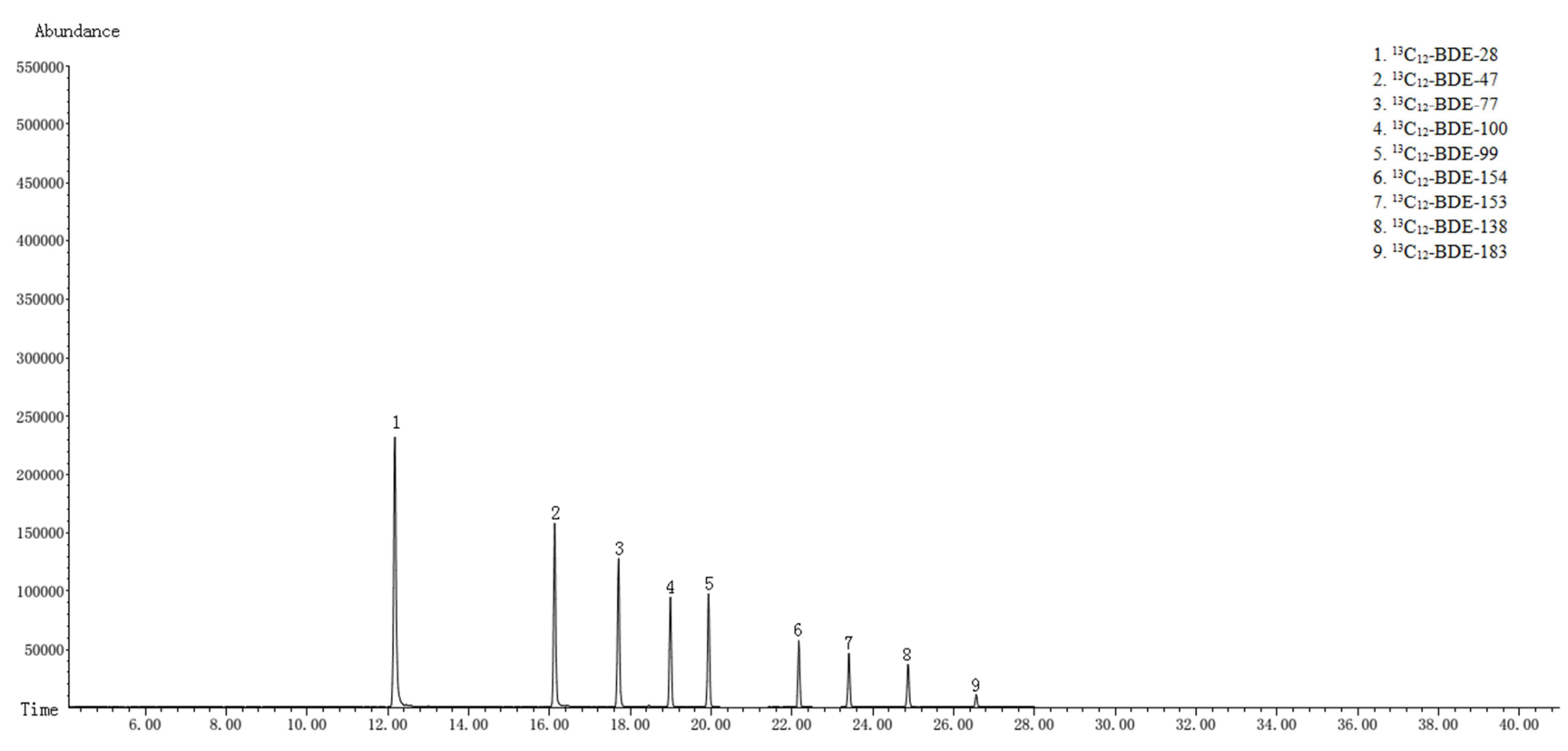

Figure 3. GC-MS chromatogram of a blank pure milk extract.

\section{Discussion}

The method to simultaneously determine eight MeO-PBDEs and fourteen PBDEs in full cream milk powders, skim milk powders, pure milks and acidophilus milks, was principally described by two methods [38, 39] as indicated above, but some important steps and crucial parameters were modified and intensified in this study. The main aspects regarding sample extraction and cleanup for sample preparations were intensified during the development of the method used.

Firstly, for sample extraction and cleanup, in contrast to the described methods [38, 39], the elution ethyl acetate was environmentally friendly reagent. As it is known, dichloromethane has strong toxicity to human and environment, and it also a potential carcinogen. Ethyl acetate was nontoxic, using it can be avoided the damage to the operating personnel.

Next, in comparison, the silica gel chromatography can only be used once. By eluting repeatedly, the Gel permeation chromatography can be used over and over again. On the other hand, GPC cleanup for lipid removal was a very important step for determination of MeO-PBDEs and PBDEs in dairy products, which was used in some literatures, but several crucial parameters in experiments were missing such as the flow rate, lipid fraction volume (F1) and fraction volume containing MeO-PBDEs and PBDEs (F2) by GPC. In contrast to the described methods (38), similarly, some details in the present study were studied to ensure the experimental feasibility. Obviously, the flow rate in GPC cleanup is a very important factor for fraction volume containing MeO-PBDEs and PBDEs collected. The high flow rate is disadvantageous for MeO-PBDEs and PBDEs separation and lipid removal, and low flow rate is time- and solvent-consuming. The flow rate of GPC in this study was optimized and $1.25 \mathrm{~mL} \min ^{-1}$ was chosen to ensure MeO-PBDEs and PBDEs separation and lipid removal.

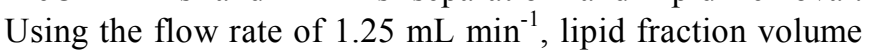
(F1) and fraction volume containing MeO-PBDEs and PBDEs (F2) collected from GPC were studied according to the below method of description. $0.4 \mathrm{~mL}$ of the mixed working standard (the concentration of BDE17, BDE28, BDE47, BDE66, BDE71, BDE85, BDE99, BDE100, BDE138, BDE153, BDE154, BDE183, BDE190 and BDE209 were $100 \mathrm{ng} \mathrm{mL} \mathrm{mL}^{-1}$, respectively, the concentration of 5-MeO-BDE47, 6-MeO-BDE47, 4'-MeO-BDE49, 2'-MeO-BDE68, 5'-MeO-BDE99, 5'-MeO-BDE100, $4^{\prime}$-MeO-BDE101 and 4'-MeO-BDE103 were $100 \mathrm{ng} \mathrm{mL}^{-1}$, respectively, the concentrations of ${ }^{13} \mathrm{C}_{12}$-labelled PBDEs 28, $47,99,100,154,153$ and 183 were $100 \mathrm{ng} \mathrm{mL}^{-1}$, respectively, and the concentration of ${ }^{13} \mathrm{C}_{12}$-labelled PBDEs 77 and 138 were $100 \mathrm{ng} \mathrm{mL}^{-1}$, respectively) was subjected to $\mathrm{GPC}$, and the eight ordinal fractions (f1, f2, f3, f4, f5, f6, f7 and f8), 25 $\mathrm{mL}$ viz. $30 \mathrm{~min}$ of time-consuming per fraction, were collected from GPC at flow rate of $1.25 \mathrm{~mL} \mathrm{~min}^{-1}$. The next experiments were conducted according to the above method. The results showed that native MeO-PBDEs and PBDEs were only detected in the fraction $\mathrm{f} 5$, and the recoveries of all these compounds were excellent. Similarly, the blank samples of full cream milk powders, skim milk powders, pure milks and acidophilus milks spiked at the same level as above mixed working standard were prepared prior to sample extractions, and the sample extractions were also carried out. The sample cleanup of GPC and following experiments were conducted according to the above method for the mixed working standard. It was also interesting that native MeO-PBDEs and PBDEs and labeled MeO-PBDEs and PBDEs were only detected in the fraction f5, and the recoveries of all these compounds ranged from $75.5 \%$ to $105.0 \%$. Moreover, beginning with fraction $\mathrm{f5}$, the fractions 
were clean and contained little lipid, which indicated the lipid removal was successful. Therefore, the first lipid fraction $(\mathrm{F} 1,100 \mathrm{~mL})$ from the $\mathrm{GPC}$ was the sum of $\mathrm{f} 1, \mathrm{f} 2, \mathrm{f} 3$ and $\mathrm{f} 4$ and discarded, and the next fraction containing MeO-PBDEs and PBDEs (F2, $100 \mathrm{~mL}$ ) was the sum of $\mathrm{f} 5$, $\mathrm{f} 6$, $\mathrm{f7}$ and $\mathrm{f8}$ and collected before following experiments in the present study.

Finally, GC and MS conditions were also very important factors for determinations of MeO-PBDEs and PBDEs, including ${ }^{13} \mathrm{C}_{12}$-labelled PBDEs. For MS acquisition, qualitative and quantitative analysis were performed using SIM. Table 1 provided further information optimized for these parameters, especially about quantitation reference for eight MeO-PBDEs and fourteen PBDEs, ensuring the operability of quantitation analysis. GC conditions were also optimized, including temperature programmed conditions and separation column. Temperature programmed conditions were optimized to ensure excellent separation efficiency of MeO-PBDEs, PBDEs and ${ }^{13} \mathrm{C}_{12}$-labelled PBDEs. Different separation columns were selected and compared to gain a more suitable separation effect, ensuring excellent experimental results.

For analysis of PBDEs and MeO-PBDEs in full cream milk powders, skim milk powders, pure milks and acidophilus milks, it was reported that PBDEs and MeO-PBDEs was present in soil and sediment at relatively high levels [37]. Six MeO-PBDEs were detected in a soil and sediment sample ranging from less than detection to $43.7 \mathrm{pg}$ g-1 dry weight per MeO-PBDE, and thirty-two PBDEs in the same soil and sediment ranging from less than detection to $1249 \mathrm{pg}$ g-1 dry weight per PBDE [37].

On the basis of those reported results, MeO-PBDEs and PBDEs was possible to be found in dairy products, which was considered to be migrated from contaminated water, soil and sediment. So it is reasonable to conclude that the MeO-PBDEs and PBDEs contamination in dairy products from China should be thoroughly investigated. Although all dairy products in this study have been fortunately proved to be $\mathrm{MeO}-\mathrm{PBDEs}$ and PBDEs negative, considering the direct links between the safety of dairy products and environmental pollution, contaminations and causes of $\mathrm{MeO}-\mathrm{PBDEs}$ as novel pollutants and PBDEs in dairy products from China still need to be investigated in the future.

\section{Conclusion}

In this paper, A new isotopic dilution GC-MS method was firstly developed to simultaneously determine fourteen PBDEs and eight MeO-PBDEs in dairy products (full cream milk powder, skim milk powder, pure milk and acidophilus milk). Solvent extraction, gel permeation chromatography (GPC) and silica gel column cleanup were used, some important steps and crucial parameters were modified and intensified compared with other literatures. Besides, the conditions of GC and MS were also optimized. The limits of quantitation values of $1-50,4-20 \mu \mathrm{g} \mathrm{kg}^{-1}$ in full cream milk powders, skim milk powders, pure milks and acidophilus milks were calculated for PBDEs and MeO-PBDEs. In addition, good repeatability and accuracy of the whole method were achieved. Therefore, the method was successfully applied to determine methoxylated polybrominated diphenyl ethers and polybrominated diphenyl ethers in dairy products.

\section{References}

[1] De Wit, C.A, An overview of brominated flame retardants in the environment, Chemosphere, 46 (2002) 583-624.

[2] Watanabe, I., Sakai, S.I, Environmental release and behavior of brominated flame retardants, Environment International, 29 (2003) 665-682.

[3] M. Alaee, P. Arias, A. Sjodin, A. Bergman, An overview of commercially used brominated flame retardants, their applications, their use patterns in different countries/regions and possible modes of release, Environ. Int. 29 (2003) 683689.

[4] R. Alcock, A.J. Sweetman, K. Prevedouros, K.C. Jones, Understanding levels and trends of BDE-47 in the UK and North America: an assessment of principal reservoirs and source inputs, Environ. Int. 29 (2003) 691-698.

[5] B.H. Wilford, T. Harner, J. Zhu, M. Shoeib, K.C. Jones, Passive sampling survey of polybrominated diphenyl ether flame retardants in indoor and outdoor air in Ottawa, Canada: implications for sources and exposure, Environ. Sci. Technol. 38 (2004) 5312-5318.

[6] H. M. Stapleton, N. G. Dodder, J. H. Offenberg, M. M Schantz, S. A. Wise, Polybrominated diphenyl ethers in house dust and clothes dryer lint, Environ. Sci. Technol. 39 (2005) 925-931.

[7] A. Kirkegaard, A. Bignert, U. Sellstrom, M. Olsson, B. Jansson, C.A. de Wit, Polybrominated diphenyl ethers (PBDEs) and their methoxylated derivates in pike from Swedish waters with emphasis on temporal trends, 1967-2000, Environ. Pollut. 130 (2004) 187-198.

[8] Sihem Ben Hassinea, Walid Ben Ameura, Ethel Eljarratb, Damià Barcelób, Soufiane Touila, Mohamed Ridha Drissa, Methoxylated polybrominated diphenyl ethers (MeO-PBDE) in human milk from Bizerte, Tunisia, Environmental research 138 (2015) 32-37.

[9] D. Handayani, R. A. Edrada, P. Proksch, V. Wray, L. Witte, R. W. Van Soest, Four new bioactive polybrominated diphenyl ethers of the sponge Dysidea herbacea from West Sumatra, Indonesia, Journal of Natural Products, 60 (1997) 1313-1316.

[10] Wen-Long Lia, Hong Qia, Wan-Li Maa, Li-Yan Liua, Zhi Zhangb, Mohammed O.A. Mohammeda, Wei-Wei Songa, Zifeng Zhanga, Yi-Fan Lia, Brominated flame retardants in Chinese air before and after the phase out of polybrominated diphenyl ethers, Atmospheric Environment, 117 (2015) 156161.

[11] L. Weijs, S. Losada, K. Das, L. Roosens, P.J. Reijnders, J.F. Santos, H. Neels, R. Blust, A. Covaci, Biomagnification of naturally-produced methoxylated polybrominated diphenyl ethers (MeO-PBDEs) in harbour seals and harbour porpoises from the southern North Sea, Environment Iinternational, 35 (2009) 893-899. 
[12] B.C. Kelly, M.G. Ikonomou, J.D. Blair, FAPC. Gobas, Hydroxylated and methoxylated polybrominated diphenyl ethers in a Canadian Arctic marine food web, Environmental Science \& Technology, 42 (2008) 7069-7077.

[13] C.L. Feng, Y.P. Xu, Y. He, Q. Luo, J.M. Zha, Z.J. Wang, Debrominated and methoxylated polybrominated diphenyl ether metabolites in rainbow trout (Oncorhynchus mykiss) after exposure to decabromodiphenyl ether, Journal of Environmental Sciences, 22 (2010) 1425-1434.

[14] P. Haglund, D.R. Zook, H.R. Buser, J. Hu, Identification and quantification of polybrominated diphenyl ethers and methoxy-polybrominated diphenyl ethers in Baltic biota, Environmental Science \& Technology, 31 (1997) 3281-3287.

[15] U. Nordlof, B. Helander, A. Bignert, L. Asplund, Levels of brominated flame retardants and methoxylated polybrominated diphenyl ethers in eggs of white-tailed sea eagles breeding in different regions of Sweden, Science of the Total Environment, 409 (2010) 238-246.

[16] S. Losada, F.J. Santos, A. Covaci, M.T. Galceran, Gas chromatography-ion trap tandem mass spectrometry method for the analysis of methoxylated polybrominated diphenyl ethers in fish, Journal of Chromatography A, 1217 (2010) 5253-5260.

[17] W.B. Ameur, S.B. Hassine, E. Eljarrat, Y.E. Megdiche, S. Trabelsi, B. Hammami, D. Barcelo, M.R. Driss, Polybrominated diphenyl ethers and their methoxylated analogs in mullet (Mugil cephalus) and sea bass (Dicentrarchus labrax) from Bizerte Lagoon, Tunisia, Marine Environmental Research, 72 (2011) 258-264.

[18] J.T. Sun, J.Y. Liu, Q. Liu, G.B. Qu, T. Ruan, G.B. Jiang, Sample preparation method for the speciation of polybrominated diphenyl ethers and their methoxylated and hydroxylated analogues in diverse environmental matrices, Talanta, 88 (2012) 669-676.

[19] K. Lofstrand, X.T. Liu, D. Lindqvist, S. Jensen, L. Asplund, Seasonal variations of hydroxylated and methoxylated brominated diphenyl ethers in blue mussels from the Baltic Sea, Chemosphere, 84 (2011) 527-532.

[20] G.B. Kim, H.M. Stapleton, PBDEs, methoxylated PBDEs and HBCDs in Japanese common squid (Todarodes pacificus) from Korean offshore waters, Marine Pollution Bulletin, 60 (2010) 935-940.

[21] A. Rotander, B. van Bavel, F. Riget, G.A. Auðunsson, A. Polder, G.W. Gabrielsen, G. Víkingsson, B. Mikkelsen, M. Dam, Methoxylated polybrominated diphenyl ethers (MeO-PBDEs) are major contributors to the persistent organobromine load in sub-Arctic and Arctic marine mammals, 1986-2009, Science of the Total Environment, 416 (2012) $482-489$.

[22] P.O. Darnerud, G.S. Eriksen, T. Johannesson, P.B. Larsen, M. Viluksela, Polybrominated diphenyl ethers: occurrence, dietary exposure and toxicology, Environmental Health Perspectives, 109 (2001) 49-68.

[23] Liqin Yua, Zhihua Hanb, Chunsheng Liu, A review on the effects of PBDEs on thyroid and reproduction systems in fish, General and Comparative Endocrinology, 219 (2015) 64-73.

[24] P. Eriksson, E. Jakobsson, A. Fredriksson, Brominated flame retardants: a novel class of developmental neurotoxicants in our environment, Environmental Health Perspectives, 109 (2001) 903-908.
[25] H. Viberg, A. Fredriksson, P. Eriksson, Neonatal exposure to the brominated flame retardant 2,2',4,4',5-pentabromodiphenyl ether causes altered susceptibility in the cholinergic transmitter system in the adult mouse, Toxicology Sciences, 67 (2002) 104-107.

[26] H. Viberg, A. Fredriksson, P. Eriksson, Neonatal exposure to polybrominated diphenyl ether (PBDE 153) disrupts spontaneous behaviour, impairs learning and memeory, and decreases hippocampal cholinergic receptors in adult mice, Toxicology and applied pharmacology, 192 (2003) 95-106.

[27] T. Zhou, M.M. Taylor, M.J. DeVito, K.M. Crofton, Developmental exposure to brominated diphenyl ethers results in thyroid hormone disruption, Toxicology Sciences, 66 (2002) 105-116.

[28] I. Branchi, E. Alleva, L.G. Costa, Effects of perinatal exposure to a polybrominated diphenyl ether (PBDE-99) on mouse neurobehavioural development, Neurotoxicology, 23 (2002) 375-384.

[29] I. Branchi, F. Capone, E. Alleva, L.G. Costa, Polybrominated diphenyl ethers: neurobehavioral effects following developmental exposure, Neurotoxicology, 24 (2003) 449-462.

[30] T. Reistad, F. Fonnum, E. Mariussen, Neurotoxicity of the pentabrominated diphenyl ether mixture, DE-71, and hexabromocyclododecane (HBCD) in rat cerebellar granule cells in vitro, Archives of Toxicology, 80 (2006) 785-796.

[31] F. Madia, G. Giordano, V. Fattori, A. Vitalone, I. Branchi, F. Capone, L.G. Costa, Differential in vitro neurotoxicity of the flame retardant PBDE-99 and of the PCB Aroclor 1254 in human astrocytoma cells, Toxicology Letters, 154 (2004) 1121.

[32] X.Z. Hu, Y. Xu, D.C. Hu, Y. Hui, F.X. Yang, Apoptosis induction on human hepatoma cells Hep G2 of decabrominated diphenyl ether (PBDE-209), Toxicol. Lett. 171 (2007) 19-28.

[33] Y.H. He, M.B. Murphy, R.M.K. Yu, M.H.W. Lam, M. Hecker, J.P. Giesy, R.S.S. Wu, P.K.S. Lam, Effects of 20 PBDE metabolites on steroidogenesis in the H295R cell line, Toxicol. Lett. 176 (2008) 230-238.

[34] R.F. Song, Y.H. He, M.B. Murphy, L.W.Y. Yeung, R.M.K. Yu, M.H.W. Lam, P.K.S. Lam, M. Hecker, J.P. Giesy, S.S. WuRudolf, W.B. Zhang, G.Y. Sheng, J.M. Fu, Effects of fifteen PBDE metabolites, DE71, DE79 and TBBPA on steroidogenesis in the H295R cell line, Chemosphere 71 (2008) $1888-1894$.

[35] X.Z. Hu, D.C. Hu, Q. Song, J. Li, P. Wang, S.F. Guo, Analysis and occurrences of perfluorinated compounds in agricultural and aquatic products and water from China, J. Liq. Chromatogr. R. T. 34 (2011) 2196-2210.

[36] X.Z. Hu, D.C. Hu, Q. Song, J. Li, P. Wang, Determinations of hexabromocyclododecane (HBCD) isomers in channel catfish, crayfish, hen eggs and fish feeds from China by isotopic dilution LC-MS/MS, Chemosphere 82 (2011) 698-707.

[37] S. Lacorte, M.G. Ikonomou, M. Fischer, A comprehensive gas chromatography coupled to high resolution mass spectrometry based method for the determination of polybrominated diphenyl ethers and their hydroxylated and methoxylated metabolites in environmental samples; Journal of Chromatography A, 1217 (2010) 337-347. 
[38] Kato Yoshihisa, Okada Syohei, Atobe Kazutaka, Endo Tetsuya, Matsubara Futoshi, Oguma Takayoshi and Haraguchi Koichi, Simultaneous Determination by APCI-LC/MS/MS of Hydroxylated and Methoxylated Polybrominated Diphenyl Ethers Found in Marine Biota, Anal. Chem, 81 (2009) 59425948.
[39] Shi Zhixiong, Jiao Yang, Hu Yue, Sun Zhiwei, Zhou Xianqing, Feng Jinfang, Li Jingguang, Wu Yongning, Levels of tetrabromobisphenol A, hexabromocyclododecanes and polybrominated diphenyl ethers in human milk from the general population in Beijing, China, Science of the Total Environment, 10-18 (2013) 452-453. 